\title{
Offset Tracing in Hall Sensors by Integrated Temperature Coefficient Determination
}

\author{
$\underline{\text { Markus Stahl-Offergeld }}^{1}$, Daniel Cichon ${ }^{1}$, Hans-Peter Hohe ${ }^{1}$, Andreas Schütze $^{2}$ \\ ${ }^{1}$ Fraunhofer Institut Integrierte Schaltungen (IIS), Dept. IC Design Mixed Signal, \\ Microsystems Technology, Erlangen, Germany \\ ${ }^{2}$ Saarland University, Lab for Measurement Technology, Saarbrücken, Germany
}

Tel: +49(0)9131/776-4674, Fax: +49(0)9131/776-499, markus.stahl-offergeld@iis.fraunhofer.de

\begin{abstract}
Hall sensors in CMOS technologies are ever present. Modern sensors are able to measure all three orthogonal components of the magnetic flux density at approximately one point [1]. Unfortunately Hall sensors have comparative high offsets, but there are a number of known techniques to reduce the offset. The best known are the spinning current technique [2] and orthogonal coupling [3]. Using these methods reduces the offset by several magnitudes, but a residual offset always remains. Additionally, this residual offset has a non-linear temperature dependency. The compensation of the residual offset can improve the sensors' performance. Up to now it has been necessary to measure the offset at different temperatures during the volume test. This takes a lot of time and, hence, costs money. With this proposed integrated temperature coefficient determination and a special algorithm, it is possible to trace the offset across the whole temperature range with only one required start-up point which can be measured easily and cheaply during the volume test.
\end{abstract}

\section{Fundamental idea}

Using an integrated heating system allows the chip to heat up by itself [4]. This temperature difference can be used to calculate the offset temperature coefficient (OTC) of the sensor. By measuring several OTCs at different temperatures, the offset characteristics across temperature could be approximated by linking up small straight lines with the according slope (OTC) as shown in figure 1.

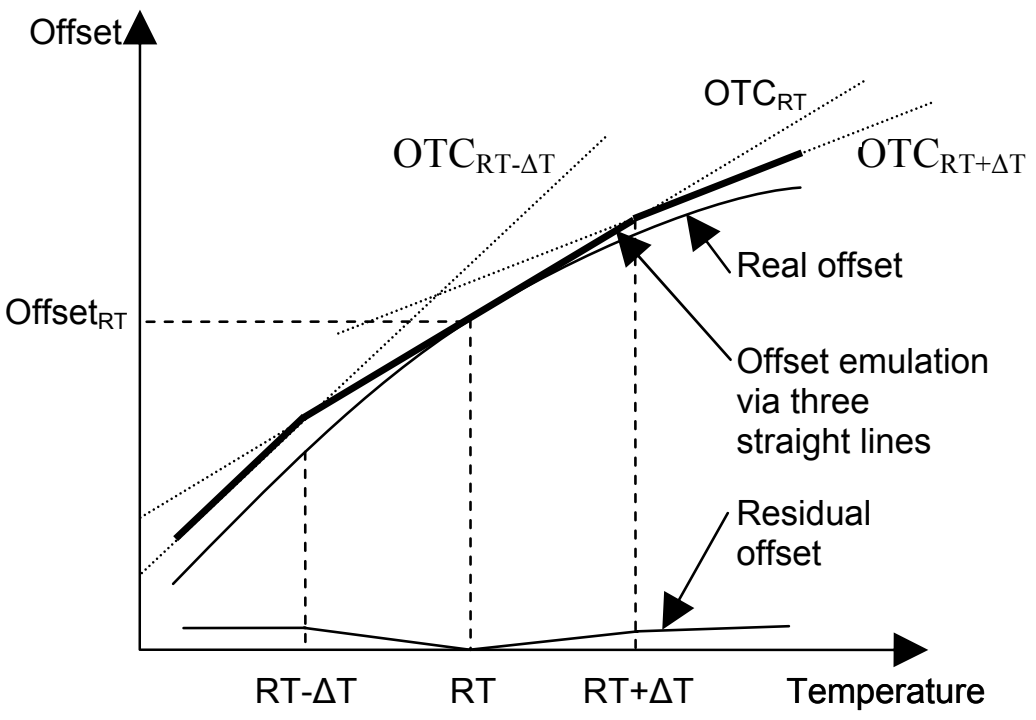

Figure 1: Fundamental idea of "offset tracing"

If the chip is used for the first time, only the offset (offset $t_{R T}$ ) and the offset temperature coefficient $\left(O T C_{R T}\right)$ at room temperature (RT) are already known from the volume test. With this information, only a simple 
straight line compensation for the offset across temperature is possible. If the ambient temperature changes, the chip could measure several OTCs at different predefined temperatures. These OTC's are stored in a table on the chip. The more OTCs are known, the better the offset can be reduced. If, during the chip's lifetime, all OTCs are noted, the offset across temperature can be traced almost exactly. In figure 2 the offset reduction was achieved with three OTCs $\left(\mathrm{OTC}_{\mathrm{RT}}, \mathrm{OTC}_{\mathrm{RT}+\Delta \mathrm{T}}, \mathrm{OTC}_{\mathrm{RT}-\Delta \mathrm{T}}\right)$

\section{Calculation of the offset temperature coefficient}

The calculation of the OTC, even with an external magnetic field can be done with the following considerations. The output voltage of a Hall sensor can be described by

$u_{\text {out }}=S_{A}(T) \cdot B_{\perp}+U_{\text {off }}(T)$

where $S_{A}(T)$ is the sensitivity of the sensor, $B_{\perp}$ is the magnetic flux density, perpendicular to the sensor plane, and $U_{o f f}(T)$ is the temperature-dependent offset voltage. The sensitivity of a voltage-controlled Hall sensor can be calculated by

$S_{A}(T)=\mu_{n}(T) \frac{w}{l} U_{B}$

where $\mu_{n}(T)$ is the carrier mobility, $w$ and $l$ are the width and length of the sensor and $U_{B}$ is the supply voltage. Because the carrier mobility is temperature-dependent, also is the sensitivity. Measuring two output voltages at two different temperatures (using the integrated heat source) leads to the following two equations

$u_{\text {out } 1}=S_{A}\left(T_{1}\right) \cdot B_{1 \perp}+U_{\text {off }}\left(T_{1}\right)$ and

$u_{\text {out } 2}=S_{A}\left(T_{2}\right) \cdot B_{2 \perp}+U_{\text {off }}\left(T_{2}\right)$.

In case of a constant magnetic flux density, $B_{1 \perp}$ equals $B_{2 \perp}$. Now the magnetic flux density can be eliminated and the offset at temperature $T_{2}$ can be calculated by

$U_{\text {off }}\left(T_{2}\right)=u_{\text {out } 2}-\frac{u_{\text {out } 1}-U_{\text {off }}\left(T_{1}\right)}{S_{A}\left(T_{1}\right)} S_{A}\left(T_{2}\right)$.

For small temperature differences the offset can be represented by a Taylor series, cut off after the first element. Thus we get the following linearization in the operating point at temperature $T_{B}$.

$U_{\text {off }}\left(T_{x}\right)=\left.\frac{d}{d T} U_{o f f}(T)\right|_{T=T_{B}} \cdot\left(T_{x}-T_{B}\right)+U_{o f f}\left(T_{B}\right)$.

The term $\left.\frac{d}{d T} U_{o f f}(T)\right|_{T=T_{B}}$ will be labelled as the offset temperature coefficient $O T C\left(T_{B}\right)$. Now the offset at temperature $T_{2}$ can be calculated by

$U_{\text {off }}\left(T_{2}\right)=\operatorname{OTC}\left(T_{1}\right) \cdot\left(T_{2}-T_{1}\right)+U_{\text {off }}\left(T_{1}\right)$.

Inserted in equation 6 this leads to

$\operatorname{OTC}\left(T_{1}\right) \cdot\left(T_{2}-T_{1}\right)+U_{\text {off }}\left(T_{1}\right)=u_{\text {out } 2}-\frac{u_{\text {out } 1}-U_{\text {off }}\left(T_{1}\right)}{S_{A}\left(T_{1}\right)} S_{A}\left(T_{2}\right)$

dissolved to $\operatorname{OTC}\left(T_{1}\right)$ leads to

$\operatorname{OTC}\left(T_{1}\right)=\frac{u_{\text {out } 2}-u_{\text {out } 1}}{T_{2}-T_{1}}+\frac{\left[u_{\text {out } 1}-U_{\text {off }}\left(T_{1}\right)\right] \cdot\left[1-\frac{S_{A}\left(T_{2}\right)}{S_{A}\left(T_{1}\right)}\right]}{T_{2}-T_{1}}$

If no external magnetic field is present, the offset at temperature $T_{1}\left(U_{\text {off }}\left(T_{1}\right)\right)$ equals the output voltage $\left(u_{\text {out } 1}\right)$ and thus the second term drops out. If an external magnetic field is present, and the offset at temperature $T_{1}$ and the sensitivities at temperatures $T_{2}$ and $T_{1}$ are known, the second term called 
correction term can be calculated. Up to now we have used an exponential function to calculate the sensitivities, but in future we will use the sensitivities measured via integrated coils [5].

\section{Detection of a changing external magnetic field}

During the derivation of the offset temperature coefficient we have assumed that the magnetic flux density stays constant during the measurements. But in a real application this assumption has to be monitored. To do so, we use more than two measurements to calculate the OTC. Figure 2 shows a diagram with five, during the heating-up sequence, measured values.

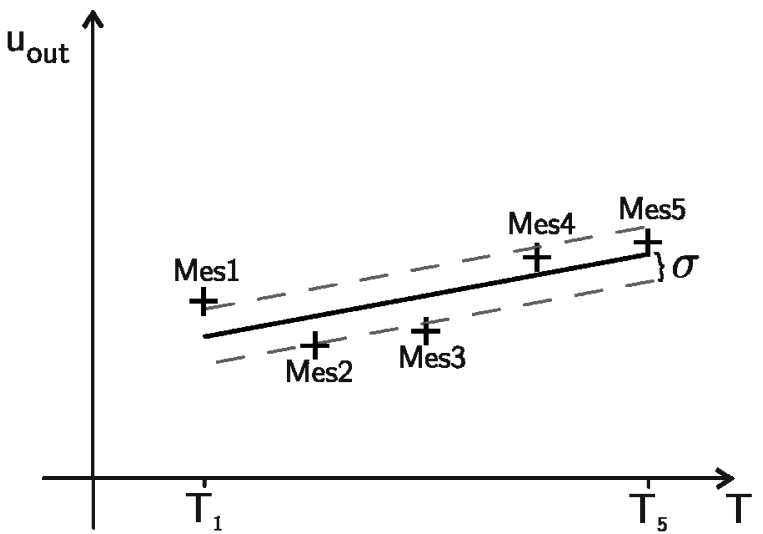

Figure 2: Five measured values during the heating-up sequence.

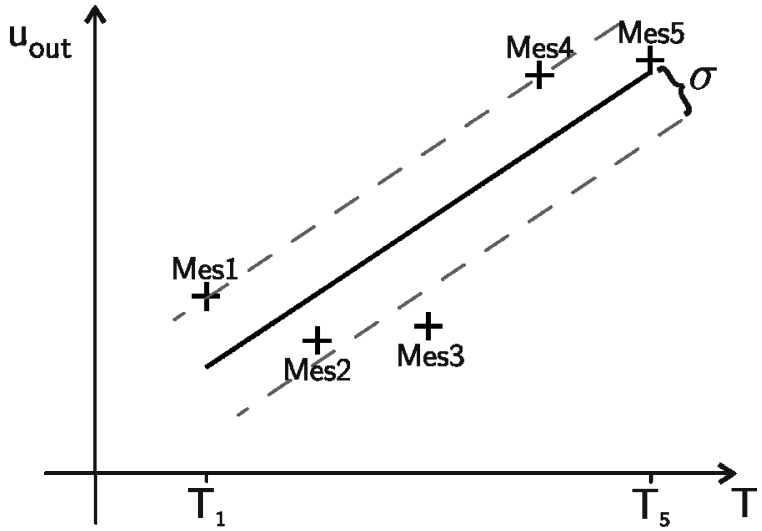

Figure 3: Changing magnetic field during the Measurement.

The first term of equation 9 (the gradient) can be determined by using linear regression. The correction term from equation 9 can be determined by using the gradient of the fitted line to calculate the offset value at $T_{1}$. Now the OTC can easily be calculated. By keeping an eye on the standard deviation $\sigma$, a change in the external magnetic field during these five measurements can be detected. Figure 3 shows five measurements with changing external magnetic field and, in consequence, with a bigger standard deviation. Thus, changes in the magnetic flux density of less than $1 \mu \mathrm{T}$ can be detected.

\section{Realization of the test chip}

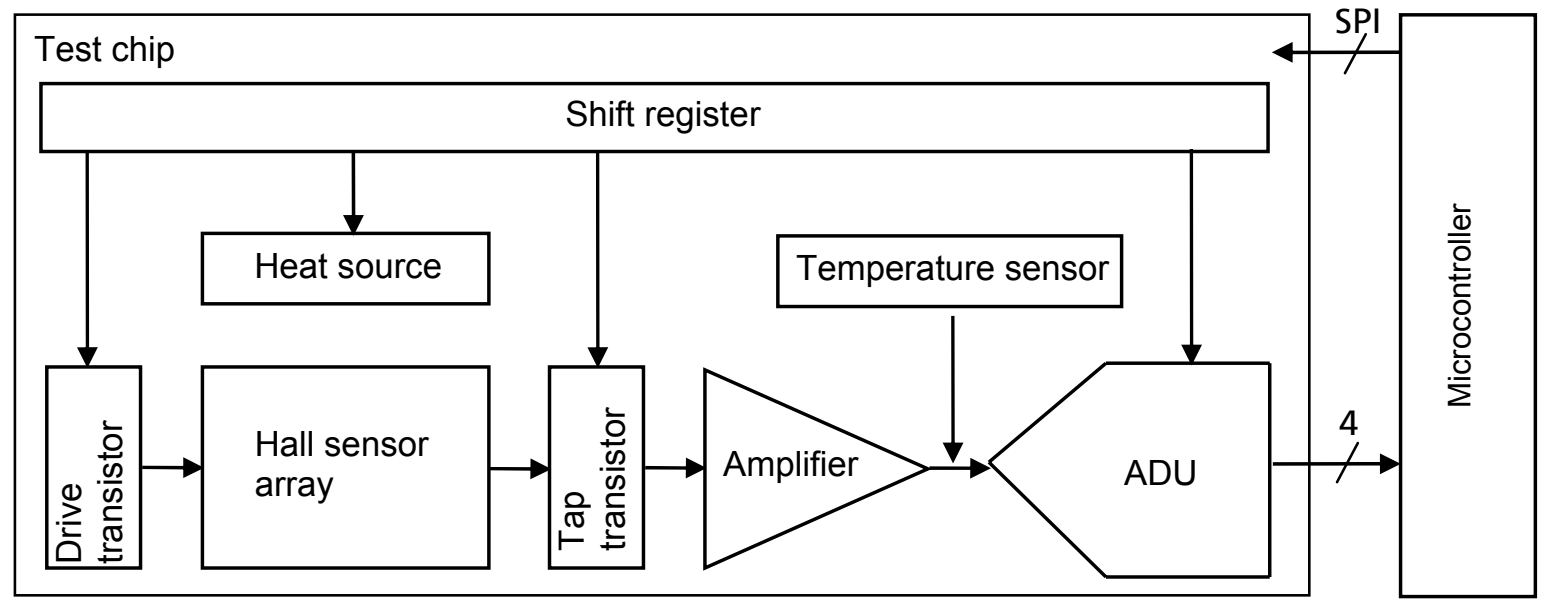

Figure 4: Block diagram of the test chip and the microcontroller

To verify the correct functionality of the offset tracing by integrated temperature coefficient determination, a microcontroller and a special test chip are used. We build the chip on a standard CMOS technology which includes an integrated heat source with two power levels realised with an n-well, nine three- 
dimensional Hall sensors, a temperature sensor, a bipolar amplifier and a four-bit delta sigma converter. All established offset reduction techniques for the sensors such as spinning current, and orthogonal coupling, as well as for the analogue signal processing such as the chopper technique, are used. The chip is controlled by the microcontroller via SPI interface. Additionally, the controller has to calculate the offset temperature coefficients. A simple block diagram of the microcontroller and the test chip is shown in figure 4 .

Because the chip has only one analogue signal processing path, the sensor and temperature signal cannot be measured at the same time, as shown in figure 5 .

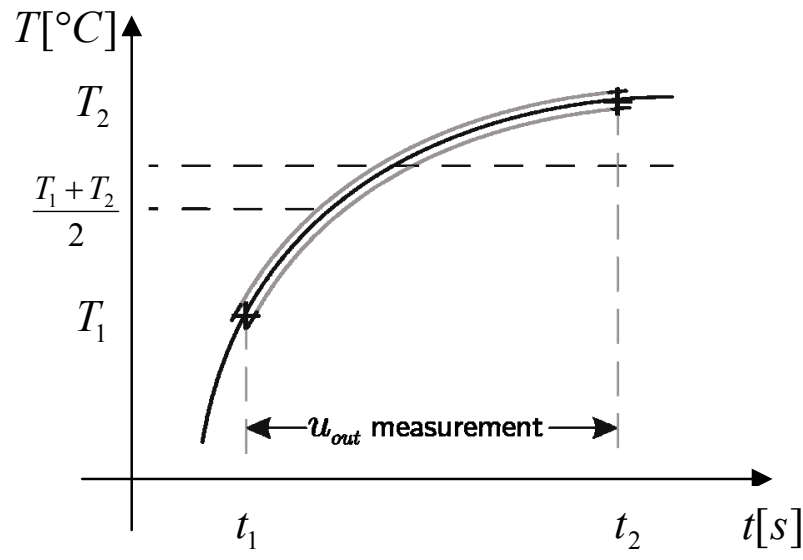

Figure 5: Temperature characteristics during the measurement

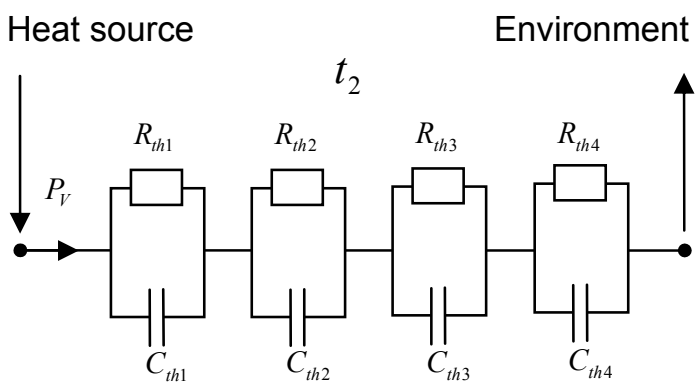

Figure 6: Thermal equivalent circuit of the sensor module

Taking two temperature measurements, one before and one after the sensor signal, and taking the arithmetic mean, still leads to incorrect OTCs. To solve this problem, we described the thermal behaviour of the sensor module with an electrical circuit like the ones used in power electronics [6], as shown in figure 6. $\mathrm{P}_{\mathrm{V}}$ is the heat flow created with the integrated heat source; $R_{t h}$ and $C_{t h}$ are thermal resistances and capacities and $\Delta \vartheta_{n}$ is the excess temperature. If at the beginning $(\mathrm{t}=0)$ the circuit is in a steady state and a constant heat flow is generated by the integrated heat source, the equation of each RC element, according to figure 6 , can be calculated by

$\Delta \vartheta_{n}=P_{V} R_{t h, n}\left(1-e^{-\frac{t}{R_{t h, n} \cdot C_{t h, n}}}\right)$.

Adding together all four excess temperatures will lead to the overall excess temperature of the sensor. By switching on the integrated heat source, the thermal impedances could be defined by analysing the step response of the system, as shown in figure 7 . The results are shown in table 1.

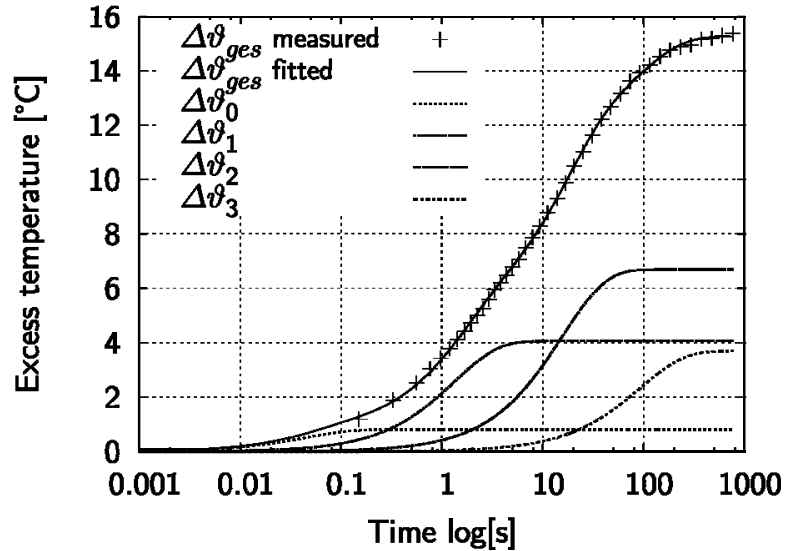

Figure 7: Thermal step response

\begin{tabular}{|c|c|c|c|c|}
\hline & $\mathrm{n}=0$ & $\mathrm{n}=1$ & $\mathrm{n}=2$ & $\mathrm{n}=3$ \\
\hline$R_{t h, n}\left[\frac{K}{W}\right]$ & 8,568 & 40,681 & 67,221 & 36,148 \\
\hline$C_{t h, n}\left[\frac{J}{K}\right]$ & 0.0053 & 0.0338 & 0.237 & 2.62 \\
\hline$\tau[m s]$ & 45.45 & 1375 & 15960 & 94770 \\
\hline
\end{tabular}

Table 1: RC values for the equivalent circuit 
With this thermal model the mean temperature during the output signal measurement can be calculated by

$$
\frac{1}{t_{2}-t_{1}} \int_{t_{1}}^{t_{2}} \Delta \vartheta(t) d t \text {. }
$$

Now the calculated mean temperature and the measured output voltage of the sensor are synchronised. This way the determined OTCs match the measured offset characteristics.

\section{Measurements}

To analyse the accuracy we achieve, we tested several sensors in a climatic chamber with temperatures between $-25^{\circ} \mathrm{C}$ and $125^{\circ} \mathrm{C}$. To generate constant and homogeneous magnetic flux densities for all three orthogonal axes at the spot of the sensors, a Helmholtz coil triple was used. With these coils magnetic flux densities of up to $10 \mathrm{mT}$ could be generated. To guarantee constant flux densities even across temperature, the coils current was controlled by a Keithley 2420 source measurement unit. The measurements started at $40^{\circ} \mathrm{C}$, then cooled down to $-25^{\circ} \mathrm{C}$, heated up again to $125^{\circ} \mathrm{C}$ and then cooled down afresh to $40^{\circ} \mathrm{C}$. This temperature cycle took about three hours. During this time, the controller continuously calculated the OTCs, checked whether the external magnetic field stayed constant and if so, used the OTCs, filtered them and stored the filtered value in a table. The reference offset measurement was taken without an external magnetic field and without offset temperature coefficient determination.

A first measurement was taken on a sensor with a typical offset variation across temperature as shown in figure 8, grey curve. The initial value for the OTC determination was only the measured offset at $25^{\circ} \mathrm{C}$. The filtered OTCs, calculated with an external magnetic field of $4.3 \mathrm{mT}$, are shown in table 2. With these values the offset tracing leads to the emulated offset shown in figure 8, black curve. In order to show the accuracy, the deviation between measured offset and emulated offset is shown in figure 9.

\begin{tabular}{|c|r|r|r|r|r|r|r|r|r|r|r|r|r|}
\hline $\begin{array}{c}\mathrm{OTC} \\
{\left[\mu \mathrm{V} /{ }^{\circ} \mathrm{C}\right]}\end{array}$ & 456 & 431 & 350 & 326 & 285 & 269 & 261 & 228 & 244 & 163 & 187 & 179 & 187 \\
\hline $\begin{array}{c}\mathrm{T} \\
{\left[{ }^{\circ} \mathrm{C}\right]}\end{array}$ & -25 & $-12,5$ & 0 & 12,5 & 25 & 37,5 & 50 & 62,5 & 75 & 87,5 & 100 & 112.5 & 125 \\
\hline
\end{tabular}

Table 2: Offset temperature coefficients stored in the microcontroller

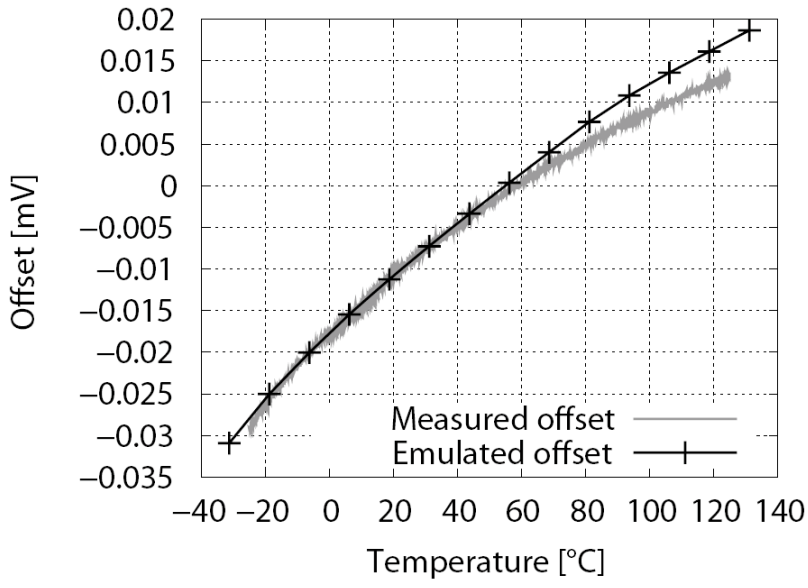

Figure 8: Measured and emulated offset across Temperature

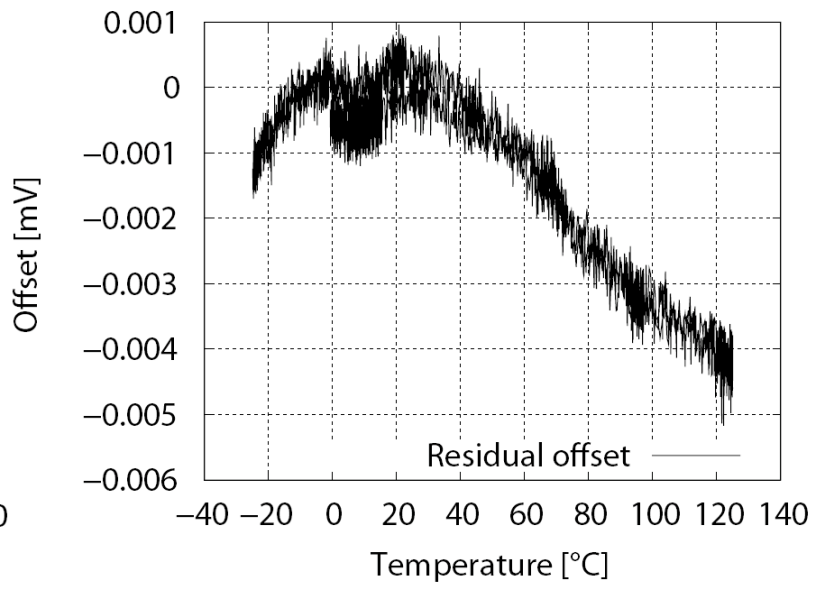

Figure 9: Offset after reduction

The original offset variation is about $45 \mu \mathrm{V}$; after the offset tracing algorithm the offset variation is reduced by more than factor 7 to only $6 \mu \mathrm{V}$. 
A second measurement was taken on a sensor with a relatively high offset variation across temperature. The measured and emulated offsets are shown in figure 10. The deviation is shown in figure 11.

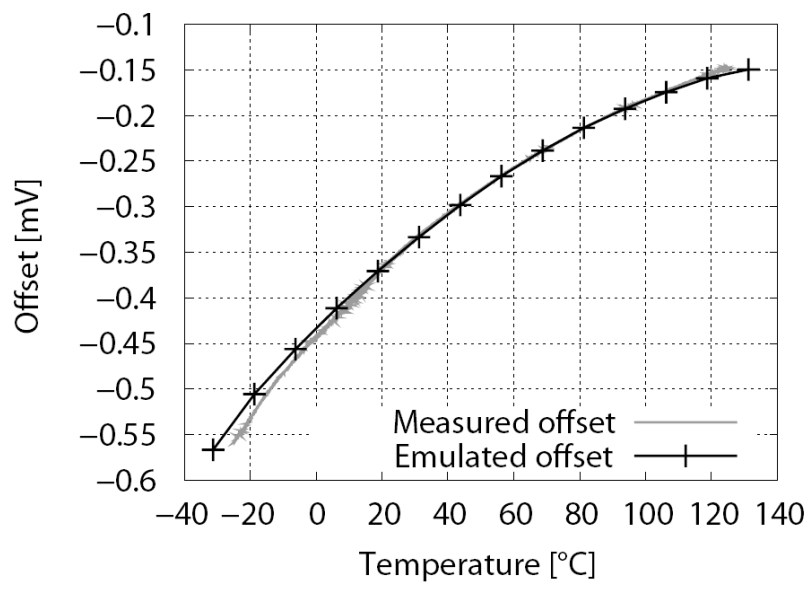

Figure 10: Measured and emulated offset across temperature of a sensor with big offset

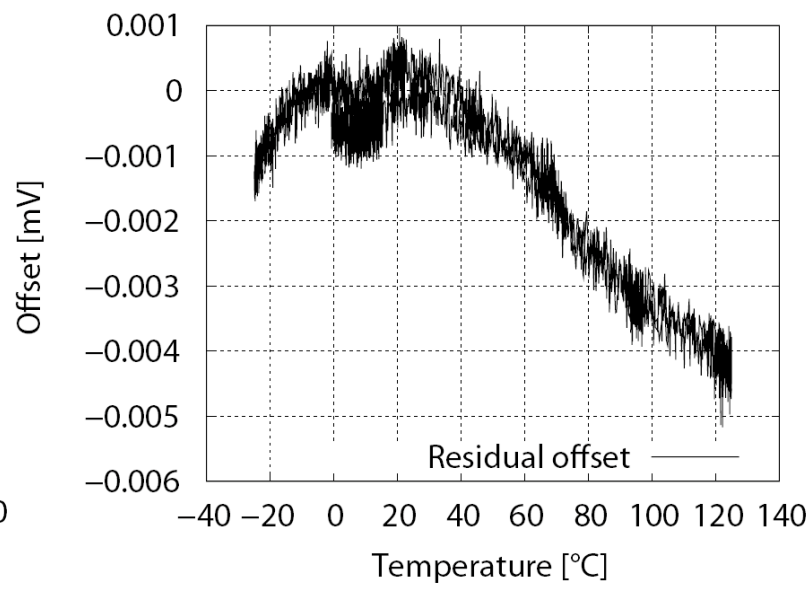

Figure 11: Offset after reduction

The original offset variation is about $400 \mu \mathrm{V}$; after the offset tracing algorithm the offset variation is reduced by more than factor 13 to only $30 \mu \mathrm{V}$.

These measurements show that using an integrated heat source gives the possibility of reducing the offset by more than a factor of 10 . Changes in the magnetic flux density of only $1 \mu \mathrm{T}$ will definitely be detected.

\section{Conclusion}

Our measurements clearly show that the offset of a Hall sensor which already uses the spinning current technique and orthogonal coupling can further be reduced by more than a factor of 10 by using integrated heat sources to calculate the offset temperature coefficients. This can even be done during measurements with an existing external magnetic field. Only one start-up point, which can easily be taken during the volume test, is necessary; the remaining offset temperature coefficient at different temperatures can be taken during the chip's lifetime.

\section{References}

[1] Ch. Schott, R.S. Popovic, "Integrated 3D Hall Magnetic field sensor", Transducers 19999, Vol.1, pp168-171

[2] A. Bellekom, "Origins of offset in conventional and spinning-current Hall plates", Delft University Press 1998

[3] P. J. A. Munter, "A Low-offset Spinning-current Hall Plate", Sensors and Actuators, A21-A23, 1990, p743-746

[4] Pöppel Josef, "Verfahren zur Temperaturkompensation bei Meßsystemen" Patent DE19703359A1 06.08.1998

[5] M. Stahl-Offergeld, H.-P. Hohe, J.Sauerer, "Integrated Sensitivity Adjustment for 3D Hall Sensors", Proceedings of the European Magnetic Sensors \& Actuators Conference 2008

[6] M.Michel, “ Leistungselektronik”, Springer, 1996, S.52-60 